01,07

\title{
Динамический термоупругий эффект в материалах с дефектной структурой
}

\author{
(C) А.Л. Глазов, К.Л. Муратиков \\ Физико-технический институт им. А.Ф. Иофрфе РАН, \\ Санкт-Петербург, Россия \\ E-mail: glazov.holo@mail.ioffe.ru \\ Поступила в Редакцию 19 января 2021 г. \\ В окончательной редакции 19 января 2021 г. \\ Принята к публикации 23 января 2021 г.
}

\begin{abstract}
В рамках термодинамического подхода определено изменение температуры при адиабатическом упругом деформировании твердых тел (термоупругий эффект) с учетом присутствия в них внутренних дефектов. Определен вклад дефектной структуры материала в формулу Кельвина при наличии в материале механических напряжений. Показано, что изменения коэффициента теплового расширения материала, обусловленные зависимостью модуля упругости и концентрации дефектов от температуры, могут иметь противоположную направленность.
\end{abstract}

Ключевые слова: термоупругость, ультразвук, фотоакустика, механические напряжения.

DOI: 10.21883/FTT.2021.05.50805.008

Изучение процессов преобразования упругой механической энергии в тепловую форму в твердых телах (термоупругий эффект) имеет длительную историю. Термоупругий эффект был открыт Джоулем и исследован Кельвином [1]. Позднее Кельвином [2] было предложено соотношение для количественного описания термоупругого эффекта. Им было установлено, что в случае упругих деформаций в адиабатических условиях изменение температуры твердых тел достаточно хорошо описывается соотношением

$$
\rho C \frac{\Delta T}{T}=-\alpha \sigma,
$$

где $T$ и $\Delta T$ - температура тела и ее изменение при нагружении соответственно; $\rho-$ плотность материала, $C$ - его удельная теплоемкость при постоянной деформации, $\alpha-$ коэффициент линейного теплового расширения, $\sigma$ - напряжени Величина $\alpha / \rho C$ часто называется термоупругой постоянной материала и характеризует степень преобразования механической энергии в тепловую форму. В соответствии с (1) относительные изменения температуры при упругом деформировании твердых тел вплоть до предельных значений напряжений в $1-2 \mathrm{GPa}$ лежат в диапазоне $\Delta T / T \sim 10^{-3}-10^{-2}$. Незначительность наблюдаемых изменений температуры под действием напряжений долгое время ограничивала интерес к термоупругому эффекту теоретическими исследованиями. Подобные исследования проводились не только в рамках термодинамики, но и с учетом особенностей поведения атомов решетки при тепловых колебаниях в присутствии упругих деформаций, включая квантовомеханический подход $[3,4]$. С их помощью удалось связать термоупругие свойства твердых тел с силовыми характеристиками взаимодействия атомов решетки и особенностями их теплового движения.
В рамках термодинамического подхода соотношение (1) легко получается при рассмотрении адиабатических процессов в твердых телах при выборе свободной энергии в виде

$$
F=K \frac{u_{l l}^{2}}{2}+\mu\left(u_{i k}-\delta_{i k} \frac{u_{l l}}{3}\right)^{2}-K \alpha_{T}\left(T-T_{0}\right) u_{l l},
$$

где $K=\lambda+\frac{2}{3} \mu$ - модуль всестороннего сжатия, $\lambda$ и $\mu-$ коэффициенты Ламэ, $\alpha_{T}$ - коэффициент теплового расширения, $\alpha_{T}=3 \alpha ; u_{i j}-$ тензор деформации.

При этом закон Кельвина в виде (1) получается, если считать упругие постоянные материала и коэффициент теплового расширения постоянными величинами. В этом случае уравнение состояния твердых тел в соответствии со свободной энергией (2) определяется хорошо известным законом Дюгамеля-Неймана. В соответствии с обозначениями работы [5] его можно записать в виде

$$
\sigma_{i j}=\lambda u_{k k} \delta_{i j}+2 \mu u_{i j}-(3 \lambda+2 \mu) \alpha \Delta T \text {. }
$$

Известно, что в действительности упругие характеристики материалов зависят от деформаций (напряжений) $[6,7]$ и от температуры $[8,9]$. Их зависимость от деформаций приводит к некоторому изменению скорости звука в материалах и ряду акустоупругих эффектов [6,7]. Несмотря на относительную слабость указанных эффектов на их основе разработан целый ряд диагностических методик, в частности, по определению внутренних напряжений в твердотельных объектах различной природы [10].

Учет зависимости упругих характеристик материалов от температуры в рамках термодинамического подхода впервые был выполнен в работе [5]. Полученные в этой и последующих работах [11-13] результаты с учетом 
возможного переменного во времени нагружения образца оказались весьма продуктивными и послужили основой для развития нового направления диагностических методик, получивших в западной литературе название Thermoelastic Stress Analysis с аббревиатурой TSA. $\mathrm{B}$ этих условиях для материалов со свободной энергией (2) соотношение Кельвина приобретает вид [5]:

$$
\begin{aligned}
\rho C \frac{\dot{T}}{T}= & -\left[\alpha+\left(\frac{v}{E^{2}} \frac{\partial E}{\partial T}-\frac{1}{E} \frac{\partial v}{\partial T}\right) s\right] \dot{s} \\
& +\left(\frac{1+v}{E^{2}} \frac{\partial E}{\partial T}-\frac{1}{E} \frac{\partial v}{\partial T}\right) \sigma_{i} \dot{\sigma}_{i}
\end{aligned}
$$

где $E$ - модуль Юнга, $v$ - коэффициент Пуассона, $s-$ первый инвариант тензора напряжений.

При выводе выражения (4) в работе [5] предполагалось, что тензор напряжений приведен к диагональному виду, поэтому под $\sigma_{i}$ понимаются диагональные компоненты тензора напряжений.

Учет зависимости упругих параметров материала от температуры открывает принципиально новые возможности использования соотношения Кельвина. Присутствие в (4) как постоянной, так и переменной во времени составляющих тензора напряжений позволяет по изменению температуры при воздействии на образец известного переменного напряжения определить и присутствующие в нем внутренние напряжения. Именно эта возможность в сочетании с развитием высокочувствительных камер для регистрации ИК излучения привела к целенаправленному проведению исследований в области TSA и практическому применению подобных методик [12-14].

Наиболее простой вид выражение (4) приобретает при нагружении образца одноосным напряжением. В этом случае можно считать, что $\sigma_{1}=s, \sigma_{2}=\sigma_{3}=0$. Тогда вместо выражения (4) получим

$$
\rho C \frac{\dot{T}}{T}=-\left(\alpha-\frac{1}{E^{2}} \frac{\partial E}{\partial T} s\right) \dot{s} .
$$

Таким образом, в соответствии с равенством (5) учет зависимости коэффициентов упругости материала от температуры приводит к закону Кельвина с некоторым эффективным значением коэффициента теплового расширения, зависящим от присутствующего в объекте напряжения.

Аналогичный термоупругий коэффициент следует учитывать и при лазерной генерации ультразвука в объектах с внутренними напряжениями, что позволяет предложить использование лазерных ультразвуковых методов и фотоакустики для диагностики остаточных напряжений. В данном случае, переменная во времени часть тензора напряжений $\sigma_{i}$, являющаяся следствием теплового возбуждения, мала по сравнению с остаточными напряжениями, так что $s \ll s$, и поэтому в случае наличия в объекте одноосного остаточного напряжения формула (5) также справедлива.
Анализ свойств различных материалов показывает, что практически для всех материалов производная $\partial E / \partial T<0$ и, следовательно, растягивающие напряжения должны всегда приводить к увеличению термоупругого коэффициента, а сжимающие - к его уменьшению. Следует отметить, что подобный результат наблюдается в большинстве TSA экспериментов $[14,15]$ и экспериментов по генерации лазерного ультразвука вблизи концов приповерхностных трещин в керамиках [16-19]. Вместе с тем в модельных экспериментах по изучению особенностей поведения лазерных ультразвуковых сигналов вблизи отверстий и отпечатков инденторов в образцах из некоторых металлов при воздействии на них одноосных напряжений были зарегистрированы и отклонения от соотношения (5) [20-22]. Правильной интерпретации результатов этих экспериментов соответствует увеличение коэффициента теплового расширения в зонах действия сжимающих напряжений и наоборот. Например, в линейном приближении фотоакустический сигнал может быть аппроксимирован функцией

$$
S=S_{0}(1+b s)
$$

где $S_{0}-$ сигнал в отсутствие напряжений, $b-$ коэффициент пропорциональности. Коэффициенты пропорциональности были измерены экспериментально для различных материалов. Из экспериментов на образцах с пробным отверстием для керамики нитрида кремния оценки дают $b_{\mathrm{Si}_{3} \mathrm{~N}_{4}}=0.01 \mathrm{MPa}^{-1}$ [19]. Для металлических образцов этот коэффициент отрицателен и равен для алюминия $b_{\mathrm{Al}}=-0.014 \mathrm{MPa}^{-1}[21,22]$, для стали $b_{\mathrm{st}}=-0.005 \mathrm{MPa}^{-1}[22]$. Такие значения не могут быть объяснены с помощью формулы (5).

Использованное в работе [5] выражение (2) соответствует модели однородной упругой среды. Вместе с тем все из исследованных лазерным ультразвуком металлических образцов были изготовлены из конструкционных сплавов, в состав которых входит значительное количество примесей и дефектов. Так, например, в сплаве алюминия Д16, исследованного в работах $[21,22]$ содержание Al составляет от 90.8 до 94.7\%. Остальное содержание составляют такие примеси как $\mathrm{Cu}, \mathrm{Mg}, \mathrm{Fe}, \mathrm{Si}$ и ряд других металлов. Наиболее типичными дефектами для металлов являются вакансии и межузельные атомы. Их характерные энергии активации для кристаллического $\mathrm{Al}$ составляют соответственно 0.54 и $2.43 \mathrm{eV}$ [23]. В реальном алюминии и его сплавах наряду с регулярным потенциалом кристаллической решетки присутствует некоторый неупорядоченный потенциал. В результате указанные энергии в реальных материалах могут иметь меньшие значения. В частности, энергия связи вакансии с дислокацией в алюминии составляет $0.181 \mathrm{eV}$ [24]. Небольшие энергии характерны и для связи вакансий с примесными атомами. Например, энергия связи вакансии с атомами меди составляет только $0.02 \mathrm{eV}$, с атомами кремния лежит в диапазоне $0.03-0.08 \mathrm{eV}$, с атомами цинка $0.02-0.03 \mathrm{eV}$, а для атомов серебра она 
имеет значение около 0.05-0.07 eV [25]. Активационная энергия для комплекса вакансия-атом водорода также относительно мала и равняется $0.16 \mathrm{eV}$ [26]. Следует для сравнения отметить, что в керамиках энергии активации дефектов имеют существенно более высокие энергии. Так, например, для исследованной нами керамики нитрида кремния типичные энергии дефектообразования составляют 2.2 и $2.75 \mathrm{eV}$ [27]. Для некоторых других керамик характерны еще более высокие значения энергии активации дефектов [28].

Для выяснения изменений соотношения Кельвина при наличии в материале образцов дефектов и примесей используем запись свободной энергии материала в виде $[29,30]$ :

$$
\begin{aligned}
F=K \frac{u_{l l}^{2}}{2} & +\mu\left(u_{i k}-\delta_{i k} \frac{u_{l l}}{3}\right)^{2} \\
& -K \alpha_{T}\left(T-T_{0}\right) u_{l l}+K \sum_{j} \Omega_{j} n_{j} u_{l l},
\end{aligned}
$$

где $\Omega_{j}$ - дилатационный параметр, характеризующий изменение объема материала при наличии в нем примесей или дефектов сорта $j, n_{j}$ - концентрация этих дефектов или примесей.

Следует отметить, что в соотношении (7) дилатационные центры учитываются в упрощенной изотропной форме. При этом концентрацию дефектов $n_{j}$ следует рассматривать как функцию от температуры и деформации (или напряжения), а знак $\Omega_{j}$ может меняться в зависимости от вида дилатационного центра. Так, например, для примесей большого радиуса или междоузлий $\Omega_{j}>0$, для примесей малого радиуса или вакансий $\Omega_{j}<0$ [29].

Далее, дифференцируя свободную энергию по деформациям, получим уравнение аналогичное закону Дюгамеля-Неймана

$$
\sigma_{i j}=\lambda u_{k k} \delta_{i j}+2 \mu u_{i j}-(3 \lambda+2 \mu) \alpha \Delta T+K \sum_{j} \Omega_{j} n_{j} .
$$

В отсутствие внутренних источников тепла и при адиабатических условиях второй закон термодинамики может быть записан как

$$
\rho C \dot{T}=T \frac{\partial \sigma_{i j}}{\partial T} \dot{u}_{i j}
$$

Подставляя сюда $\sigma_{i j}$ из (8) и выражая деформации $u_{i j}$ через напряжения $\sigma_{i j}$, получим следующее соотношение Кельвина

$$
\begin{aligned}
& \rho C \frac{\dot{T}}{T}=-\left[\alpha-\frac{1}{3} \sum_{j} \Omega_{j} \frac{\partial n_{j}}{\partial T}+\left(\frac{v}{E^{2}} \frac{\partial E}{\partial T}-\frac{1}{E} \frac{\partial v}{\partial T}\right.\right. \\
& \left.\left.-\frac{1}{3} \sum_{j} \Omega_{j} \frac{\partial^{2} n_{j}}{\partial T \partial s}\right) s\right] \dot{s}+\left(\frac{1+v}{E^{2}} \frac{\partial E}{\partial T}-\frac{1}{E} \frac{\partial v}{\partial T}\right) \sigma_{i} \dot{\sigma}_{i},
\end{aligned}
$$

Для случая одноосного нагружения образца $\sigma_{1}=s$, $\sigma_{2}=\sigma_{3}=0$ выражение (10) принимает вид

$$
\begin{aligned}
\rho C \frac{\dot{T}}{T}= & -\left(\alpha-\frac{1}{3} \sum_{j} \Omega_{j} \frac{\partial n_{j}}{\partial T}-\frac{1}{E^{2}} \frac{\partial E}{\partial T} s\right. \\
& \left.-\frac{1}{3} \sum_{j} \Omega_{j} \frac{\partial^{2} n_{j}}{\partial T \partial s} s\right) \dot{s} .
\end{aligned}
$$

Из полученного результата видно, что при наличии дефектов и действии в материале одноосного напряжения его поведение можно охарактеризовать эффективным значением коэффициента теплового расширения

$$
\alpha_{\mathrm{eff}}=\alpha-\frac{1}{3} \sum_{j} \Omega_{j} \frac{\partial n_{j}}{\partial T}-\frac{1}{E^{2}} \frac{\partial E}{\partial T} s-\frac{1}{3} \sum_{j} \Omega_{j} \frac{\partial^{2} n_{j}}{\partial T \partial s} s .
$$

Второе слагаемое в этом выражении учитывает изменение размеров тела вследствие изменения концентрации дефектов в материале с температурой. Третье слагаемое, как отмечалось, учитывает влияние напряжения на коэффициент теплового расширения бездефектного материала, и его знак определяется знаком $s$ в силу того, что $\partial E / \partial T<0$. Второе и последнее слагаемые учитывают вклад дефектной подсистемы в общий коэффициент теплового расширения при действии напряжения.

Для того, чтобы оценить вклад в коэффициент теплового расширения слагаемых с $n_{j}$ воспользуемся подходами, разработанными в работах [31-33]. В соответствии с ними вероятность деформирования атомной структуры материала в единицу времени при наличии механического напряжения $s$ определяется выражением

$$
p=\frac{1}{\tau_{0}} \exp \left(-\frac{U-\Omega s}{k_{b} T}\right)
$$

где $\tau_{0}$ - время порядка периода колебаний атома в решетке, $U$ - энергия активации процесса дефектообразования, $\Omega$ - его активационный объем, $k_{b}-$ постоянная Больцмана.

В соответствии с (13) для кинетики дефектных центров можно записать уравнение

$$
\frac{\partial n}{\partial t}+\frac{n}{\tau}=\frac{n_{0}}{\tau_{0}} \exp \left(-\frac{U-\Omega s}{k_{b} T}\right)
$$

где $n_{0}-$ концентрация потенциальных дефектов в материале, возбуждаемых активационной энергией $U$.

Если напряжение было приложено к объекту при $t=0$, то концентрация дефектов в нем к моменту времени $t$ будет определяться выражением

$$
n=\frac{n_{0}}{\tau_{0}} \int_{0}^{t} d t^{\prime} \exp \left(-\frac{t-t^{\prime}}{\tau}\right) \exp \left(-\frac{U-\Omega s}{k_{b} T}\right) .
$$

Предлагаемый подход для определения концентрации $n$ уже был использован нами для объяснения особенностей 
колебаний алюминиевых мембран при их возбуждении лазерным излучением [34]. С помощью полученного выражения можно определить знак второй производной от концентрации $n$ по температуре и напряжению в равенстве (9). Нетрудно показать, что при $U>k_{b} T$, $U>\Omega s$ ее знак всегда положительный. Поэтому в соответствии с (13) учет искажения химических связей атомов материала при действии напряжения приводит к изменению $\alpha$ противоположному по знаку $\partial E / \partial T$. Следует отметить, что второе слагаемое в равенстве (12) с первой производной по температуре от концентрации $\mathrm{n}$ также дает вклад в зависимость коэффициента теплового расширения от напряжения, но он в $k_{b} T / U$ раз меньше, чем от второй производной. Из выражения (15) также следует, что учет вклада деформационных центров в коэффициент теплового равновесия в первую очередь актуален для металлов, так как для керамик характерны более высокие значения активационных энергий.

В заключение следует отметить, что в соответствии с приведенным термодинамическим рассмотрением наличие дефектных состояний в твердых телах влияет на особенности проявления закона Кельвина как в материалах без механических напряжений, так и при их наличии. При этом в присутствии дефектов в материалах может изменяться характер проявления динамического термоупругого эффекта в зависимости от внутренних и внешних механических напряжений. Подобные особенности эффекта Кельвина в первую очередь должны быть характерны для материалов с низкими уровнями активационных энергий для дефектных состояний материала.

\section{Конфликт интересов}

Авторы заявляют, что у них нет конфликта интересов.

\section{Список литературы}

[1] W. Thompson (Lord Kelvin). Trans. Roy. Soc. Edinburgh 20, 261 (1853).

[2] W. Thompson (Lord Kelvin). Mathematical and Physical Papers. Cambridge University Press, London (1890). 592 p.

[3] В.Л. Гиляров, А.И. Слуцкер, В.П. Володин, А.И. Лайус. ФТT 40, 1548 (1998).

[4] В.Л. Гиляров, А.И. Слуцкер. ФТТ 56, 2404 (2014).

[5] A.K. Wong, R. Jones, J.G. Sparrow. J. Phys. Chem. Solids 48, 8, 749 (1987).

[6] Y.H. Pao, W. Sachse, H. Fukuoka. In: Physical Acoustics. Academic Press, N.Y. (1984). XVII. P. 62.

[7] А.Н. Гузь. Упругие волны в телах с начальными напряжениями. Наук. думка, Киев (1986). 372 с.

[8] P. Ferraro, R.B. McLellan. Met. Trans. 8A, 1563 (1977).

[9] J. Vanhellemont, A. K. Swarnakar, O. Van der Biest. ECS Transactions 64, 11, 283 (2014).

[10] В.В. Муравьев, Л.Б. Зуев, К.Л. Комаров. Скорость звука и структура сталей и сплавов. Наука, Новосибирск (1998). $184 \mathrm{c}$.
[11] R.J. Greene, E.A. Patterson, R.E. Rowlands. In: Springer Handbook of Experimental Solid Mechanics / Ed. W.N. Sharpe, Jr. Springer, Boston, MA (2008). P. 743.

[12] R. Emery, J.M. Dulieu-Barton. Compos. A 41, 12, 1729 (2010).

[13] P. Bian, X. Shao, J.L. Du. Appl. Sci. 9, 11, 2231 (2019).

[14] R.A. Tomlinson, E.J. Olden. Strain 35, 2, 49 (1999).

[15] S.J. Lin, W.A. Samad, A.A. Khaja, R.E. Rowlands. Exp. Mech. 55, 653 (2015).

[16] K.L. Muratikov, A.L. Glazov, D.N. Rose, J.E. Dumar. J. Appl. Phys. 88, 5, 2948 (2000).

[17] K.L. Muratikov, A.L. Glazov, D.N. Rose, J.E. Dumar. High Temp. High Press. 33, 285 (2001).

[18] К.Л. Муратиков, А.Л. Глазов. ЖТФ 73, 8, 90 (2003).

[19] A.L. Glazov, K. L. Muratikov. J. Phys.: Conf. Ser. 1697, $012186(2020)$.

[20] А.Л. Глазов, Н.Ф. Морозов, К.Л. Муратиков. ФТТ 58, 9, 1679 (2016).

[21] А.Л. Глазов, К.Л. Муратиков. Письма в ЖТФ 45, 17, 51 (2019).

[22] А.Л. Глазов, К.Л. Муратиков. Письма в ЖТФ 46, 23, 33 (2019).

[23] N. Sandberg, B. Magyari-Köpe, T.R. Mattsson. Phys. Rev. Lett. 89, 6, 065901 (2002).

[24] E. Clouet. Acta Mater. 54, 3543 (2006).

[25] C. Wolverton. Acta Mater. 55, 5867 (2007).

[26] G.A. Young Jr., J.R. Scully. Acta Mater. 46, 6337 (1998).

[27] S.P. Ogden, T.M. Lu, J.L. Plawsky. J. Appl. Phys. 109, 15, 152904 (2016).

[28] Y. Dong, L. Qi, J. Li, I.W. Chen. Acta Mater. 126, 438 (2017).

[29] Ф.Х. Мирзоев, В.Я. Панченко, Л.А. Шелепин. УФН 166, 1, 3 (1996).

[30] А.М. Косевич. Основы механики кристаллической решетки. Наука, М. (1972). 280 с.

[31] S.N. Zhurkov. Int. J. Fract. Mech. 1, 4, 311 (1965).

[32] С.Н. Журков. ФТТ 25, 10, 3119 (1983).

[33] H. Spikes. Friction 6, 1, 1 (2018).

[34] A.L. Glazov, K. L. Muratikov. J. Appl. Phys. 128, 095106 (2020).

Редактор Ю.Э. Китаев 